\title{
Amazon Machine Learning vs. Microsoft Azure Machine Learning as Platforms for Sentiment Analysis
}

\author{
Osama Harfoushi ${ }^{1}$ and Dana Hasan ${ }^{2}$ \\ ${ }^{1}$ Department of Business Information Technology, \\ The University of Jordan, Jordan \\ ${ }^{2}$ Microsoft Dynamics technical Consultant, \\ ebecs A DXC Technology Company, Jordan \\ o.harfoushi@ju.edu.jo
}

\begin{abstract}
Recently, there has been an increasing attention towards the use machine learning platforms notably Amazon Machine learning and Microsoft Azure Machine learning (ML) to undertake sentiment analysis. The present experimental study compared Amazon ML against Microsoft Azure ML as platforms for performing sentiment analysis. The evaluation was done $d$ using the evaluation metrics: accuracy, reliability, precision, F-score and Recall. Data was sourced from Twitter a microblogging platform. The sentiment analytics model was created based on Logistic regression analysis algorithms. Results confirmed that Microsoft Azure ML is more accurate and precise than Amazon ML as platforms for Sentiment Analysis and that Azure could be used reliably, accurately and securely to build Sentiment Analysis models for social media sites.
\end{abstract}

Key words: Sentiment Analysis, F-score, Machine Learning, Microsoft Azure, Amazon Machine Learning

\section{Introduction}

For most business enterprises, machine learning as a service (MLaaS) and sentiment analysis presents an opportunity to create working models and obtain valuable insights from predictions. MLaaS, which encompasses semi-automated and automated cloud platforms covering infrastructural issues such as model training, data pre-processing, model evaluation and prediction, can be bridged with sentiment analysis and used to undertake sentiment analysis. Through sentiment analysis, a business use natural language processing to analyse and detect sentiments or opinions in a social media text. This has been demonstrated in several studies. For example, [1] demonstrated that Machine Learning platform by the leading providers of cloud service (i.e., Microsoft Azure Machine Learning and Amazon (AWS)) can provide a platform for undertaking twitter sentiment analysis. Similarly, [2] used a machine learning based approached to analyse public opinions via social media on HPV vaccines. The study aimed at identifying a machine learning system that can be used to extract public sentiments via Twitter regarding the HPV vaccines. In a similar study, [3] used Microsoft Azure Machine Learning to undertake Twitter sentiment analysis and to create a model of machine learning classification that identifies sentiments and contents in a tweet that illustrates positive-value user contribution. Using Al-powered cognitive tools and data mining tools, [3] analysed promotional learning and other factors of social influence in the developer community. The predictive model developed in this study involved a combination of a custom-developed natural language model and a traditional supervised machine learning algorithm for identifying promotional tweets via

Received (March 23, 2018), Review Result (July 23, 2018), Accepted (August 3, 2018) 
Twitter with an accurate rate of 90 percent. Similarly, [4] presented an architecture for designing social media intelligence solutions and showcased two case studies built on this architecture. The two case studies involved the analysis of social graph influence in Facebook-influence Movie Recommendations and User-Generated Content with Sentiment Analysis on Twitter. The model involves cloud-hosted solutions in cloud providers such as Google Cloud, Microsoft Azure and Amazon AWS. Results of these studies demonstrate that machine learning platforms namely Google Cloud, Microsoft Azure, and Amazon AWS can provide a platform for undertaking sentiment analysis of social media data. In another similar study, [5] performed Twitter sentiment analysis using Microsoft Azure Machine learning and developed a model used to classify machine learning that facilitate the identification of the content and sentiments in a tweet that illustrate positive-value user contribution. However, these studies with the exception of [6] reveal little about the accuracy and reliability of these machine learning platforms and in particular Amazon Azure and Amazon ML to undertake sentiment analysis. In [6], Microsoft Azure and Amazon AWS were used to as platforms for performing sentiment analysis to analyse opinions and reviews of customers on Twitter. Results were analysed and explained in terms of the effectiveness of Microsoft Azure Amazon AWS in revealing the emotion sand polarity classifications. It was noted that Microsoft Azure performs better in the polarity classification with $65 \%$ positive tweets and 25\% negative polarity than Amazon with $45 \%$ positive tweets and 50\% negative polarity. On the contrary, from the emotions classification, Microsoft Azure was better than Amazon in the "joy" category but poorer than Amazon in the "sadness" category. Worth noting is that results of this study revealed little about the accuracy and reliability of these platforms for sentiment analysis. Informed by this gap in research, the present comparative study conducted experiments on twitter datasets with a view to evaluating using the evaluation metrics (accuracy, reliability, precision, F-score and Recall) Amazon ML and Microsoft Azure ML as platforms for performing sentiment analysis. This study extended the research conducted in a previous thesis study with updated new experiments' results [21].

\section{Literature Review}

\subsection{Sentiment Analysis and Logistics Regression}

Sentiment analysis or opinion mining is an information extraction process and natural language processes that involves the analysis of large number of documents with a view to derive the speakers' attitude or opinion. It can be used to analyse huge repository of unstructured and structured social media data to determine whether users' attitudes or opinion are neutral, negative or positive. Positive sentiments express positive review, feedback, or opinion about a product or service Sentiment. Conversely negative reviews express negative feedback, reviews or opinion about a product or service. A neutral sentiment express both positive nor negative sentiments about a product or service [7], [8] and [9].

Sentiment classification methods can be identified into three: lexicon-based approaches; hybrid approaches and machine learning approaches. The lexicon-based techniques rely on sentiment lexicon. The hybrid approaches involve a combination of machine learning and lexicon based approaches. Machine learning approaches, which is the focus of the present study use linguistic features and apply ML algorithms to solve sentiment analysis. Machine learning approaches are further categorized into supervised learning and unsupervised learning. The supervised learning approaches utilize labelled training documents while unsupervised approaches are used whenever the libelled training documents cannot be found with ease. In the present study logistic regression was used to evaluate the accuracy and reliability of Amazon ML and Microsoft Azure [10] and [11]. 
It is also important to highlight that SA enables an enormous number of applications. SA applications are increasing in several fields especially those applications based on the Web and social media. To begin with, SA improves the capabilities of recommendation system which is the type of system that filters a list of items based on users' preferences. For instance, SA works within recommendation systems by displaying the items that have positive reviews at the head of the list, followed by items that hold negative reviews. Therefore, SA will improve customer relationship management. However, as this is beneficial for businesses to display the positive reviews first to attract more customers, users might be affected if they did not spend more time looking for all the reviews.

The Logistic Regression is one of the well-known statistical linear algorithms for classification tasks. For prediction, it applies statistical analysis to predict value based on a number of variables. Classifiers building with this type of algorithm start by performing extraction for a group of weighted features, then obtain the log-odds. Afterward, linear joining is applied in which features are multiplied by the weight and combined. Moreover, classification models based Logistic Regression algorithm are viewed to be effective but for solving simple problems. Logistic regression algorithm is a statistical technique which was developed by David Cox. It is used in machine learning as an algorithm for determining the relationship between an output (dependent) categorical variable and predictors (independent) variables and in classifying tasks. It evaluates the probability by employing a logistic function [12].

\subsection{Cloud Computing Platforms: Amazon ML vs Azure ML}

Amazon launched its machine learning Amazon Web Services (AWS) in 2006. Amazon was also the first cloud provider to provide Infrastructure as a service (IaaS), enabling organizations and individuals to rent virtual computers. AWS has Elastic Cloud (EC2) that enables users to run applications by allocating the required computers. It allows for the creation of virtual machines for achieving the users' needs scalability deployment of application. It allows for the hourly computation of payment for active instance [14].

Amazon Machine Learning is one of the two machine learning services provided by Amazon. It is an automated solution to predictive analytics widely consider appropriate for use in deadline-sensitive operations. It allows the service to load data from CSV files, Amazon Redshift, Amazon RDs and other sources. It automatically performs all data preprocessing operations. Its service identifies categorical and numerical fields, and it does not require the user to select the methods used in pre-processing of data. However, its prediction capabilities only have three options: regression, multiclass classification, and binary classification. Also, it does not support unsupervised learning techniques and requires the user to select and label a target variable in a training set. Furthermore, it does not require one to be competent in machine learning methods

It is considered the most automated predictive analytics machine learning on the market. It performs all data pre-processing operations automatically. It has the service identifies with categorical and numerical fields and does not require users to choose the techniques to facilitate further pre-processing of data. It is considered the best machine learning predictive analytics for use in deadline-sensitive operations. It is capable of loading data from multiple sources, including Amazon Redshift, Amazon RDS, and CSV files. It supports various programming languages and frameworks. Users can use it to build, deploy and manage the service and applications via Microsoft global network datacentres. This cloud platform can allow users use different types of instances at an hourly rate. It has limited prediction capabilities: regression, multiclass classification and binary classification. It does not support unsupervised learning methods which means that its users must by identify a target variable in a training set to label it. Again, Amazon machine learning automatically selects machine learning methods. As such, it does not require users to be conversant of the machine learning methods [15]. 
On the other hand, Microsoft Azure infrastructure and cloud platform was released in 2010. It offers both Infrastructure as a Service (IaaS) and Platform as a Service (PaaS) delivery models. Microsoft Azure sets a powerful playground for experienced data scientists and newcomers. It has a similar roster of machine learning products to that of Amazon. Its services can be categorized into two: Bot Service and Azure Machine Learning Studio. ML Studio allows almost all operations to be completed manually, including data pre-processing, exploration, validating modelling results, and selecting methods. Approaching Azure machine learning involves a learning curve. Microsoft Azure supports graphical interface and supports multiple algorithms and about 100 methods that address anomaly detection, classification, recommendation, regression, and text analysis [13].

Moreover, Azure offer virtual servers that users can use for running applications, these virtual servers contain hardware devices such as storage, servers, and infrastructure such as the cooling system and power supply. Controlling and maintaining the virtual servers is Azure responsibility, not the users. Azure provides virtual servers based on Windows and Linux Operating Systems (OS). Additionally, Azure also provides a software environment for developers to build and run their application on the Azure infrastructure. In this case, developers do not need to be concerned about how the infrastructure is managed since the details of the infrastructure are hidden from the developers and Azure manages the installing of OS and preparing a suitable environment to run the applications in. Also, managing the environment security and setting up network configurations are Azure's responsibility. Furthermore, Azure provides a number of services that end-users can access through endpoints including, but not limited to the following: SQL database, storage service, service bus and BizTalk Service. For using these services, users are not required to install any type of software to support these services.

\section{Methodology}

This study employed a hybrid quantitative and qualitative research approach. Sentiments from twitter mining based on quantitative and qualitative identified by shaded objects are classified using machine learning algorithms. The acquisition of qualitative data involves using a customized software to collect data from Twitter as well as the users' experiences in using both platforms. Figure 1 identified the comparison elements for both Azure ML and Amazon ML platforms. Interface and Data Acquisition are compared using qualitative approach which Runtime experiment are computed using quantitative experiment method.

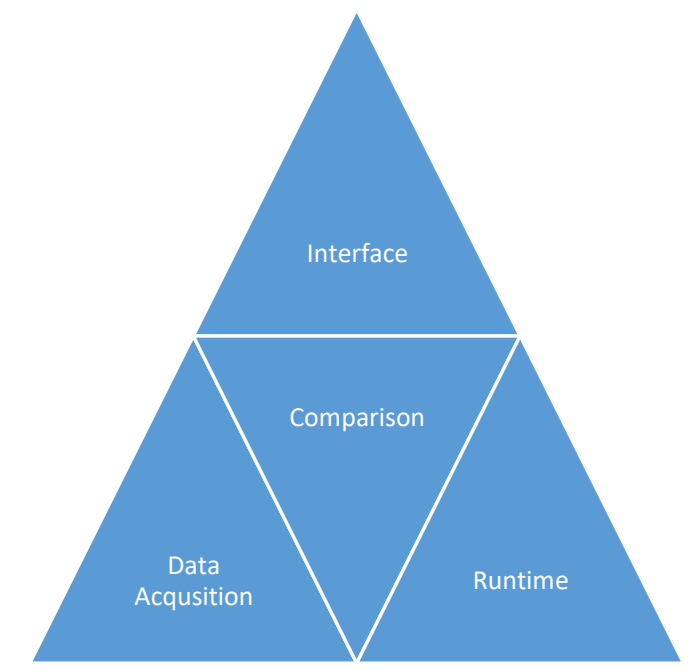

Figure 1. Platforms Comparison Criterion 
Data used in this study was collected from twitter. Twitter is a social networking website for news. It can be considered a hybrid of SMS messaging, instant messaging and email all rolled into a single simple and neat package. Data collected from twitter undertaken the following processes: data preparation and selection of attributes; pre-processing; data cleansing; and sentiment classification.

Data preparation involved manipulating data into a suitable format for further processing and analysis. This process involved routine, tedious and time-consuming data preparation non-automated processes. Selection of attributes involved selecting a subset of variables and predictors used in the model construction.

Pre-processing of data was done, which involved data tokenization; removal of stop words; POS tagging; and Steaming. Data Tokenization is used to perform separation in the sentence into words, symbols, phrases or other components called tokens. In this task, the punctuation marks are excluded for instance, full stop, comma, and question mark. Stop word removal means a collection of stop words are removed. A list of words will be filtered in the case if the words do not impact to the analysis and hold unnecessary information. Determiners words and prepositions words are both examples of stop words. On the other hand, POS tagging is for distinguishing the diverse parts of speech and assigning POS to each token such as verbs, adverbs, adjectives, and nouns. Finally, Stemming is the method that alters the form of a word to its root or base form without concerning about the word POS.

Data cleansing or data scrubbing was done to detect, correct and remove inaccurate, incomplete, incorrect, and corrupt data from table, a record set, or database and replacing the sensitive data with identification symbols that preserve the essential information without compromising its security. This was done by removing stop words and common words filtered outs after and before the natural language data was processed.

Sentiment classifications classifies sentiments within texts. It classifies sentences or documents that hold opinions towards specific entity into three classes; positive, negative or neutral. This task is challenging specifically when it deals with texts from different languages or several domains.

Bothe Azure ML and Amazon ML Sentiment Analysis Model used to analyse "Coachella tweets dataset". It was used to determine the tweets' sentiment. This was done by building both Azure and Amazon ML models, training them on how to detect the sentiment, and finally setting them as a predictive model to facilitate the detection and identifying sentiments as neutral, negative or positive.

The Sentiment Analytics models were created based on the Logistic Regression algorithm. The two models were trained using dataset (TD). This was done after subjecting the dataset to normalization, which involved getting rid of punctuations, numbers and stop words as well as removing URLs and emails from the tweets. Lastly, modifications were made to each model to make it detect sentiment.

Coachella 2015 Twitter sentiment dataset from "CrowdFlower" data mining company was used. A total of 1250 records of tweets out of 3800 records were utilized in the present study. These records were free from duplicate tweets. These records were identified into One datasets with total number of 900 records.

Evaluation matrices namely F-scores, Recall, Precision and Accuracy scores were used to evaluate the text classification tasks and the quality of SA models created on Amazon and Azure MLs. The accuracy metrics determined the correctness of the model by measuring the extent to which the prediction generated by the model was accurate. Precision determines the exactness or the closeness of the measured values to each other. The recall metric or the sensitivity metric represented the percentage of positive tweets or values predicted by the model as being positive. The F-score involved a combination of the recall and precision metrics to determine the score. The Confusion matrix (Figure 2) is used to assess the performance of both models. This Figure presents the true sentiment values 
(TPOS, TNEU, TNEG) and the false sentiment values (FPOS, FNEU, FNEG). It is used to compare the model predicted values with the actual values.

1. $\left(T_{P O S}, T_{N E U}, T_{N E G}\right)$ : represents the amount of values the model predicted correctly in each class.

2. $\left(F_{P O S}, F_{N E U}, F_{N E G}\right)$ : represents the amount of values the model predicted incorrectly in each class.

The confusion matrix is used to evaluate the model performance on each test and the evaluation metrics are calculated based on the matrix following these equations:

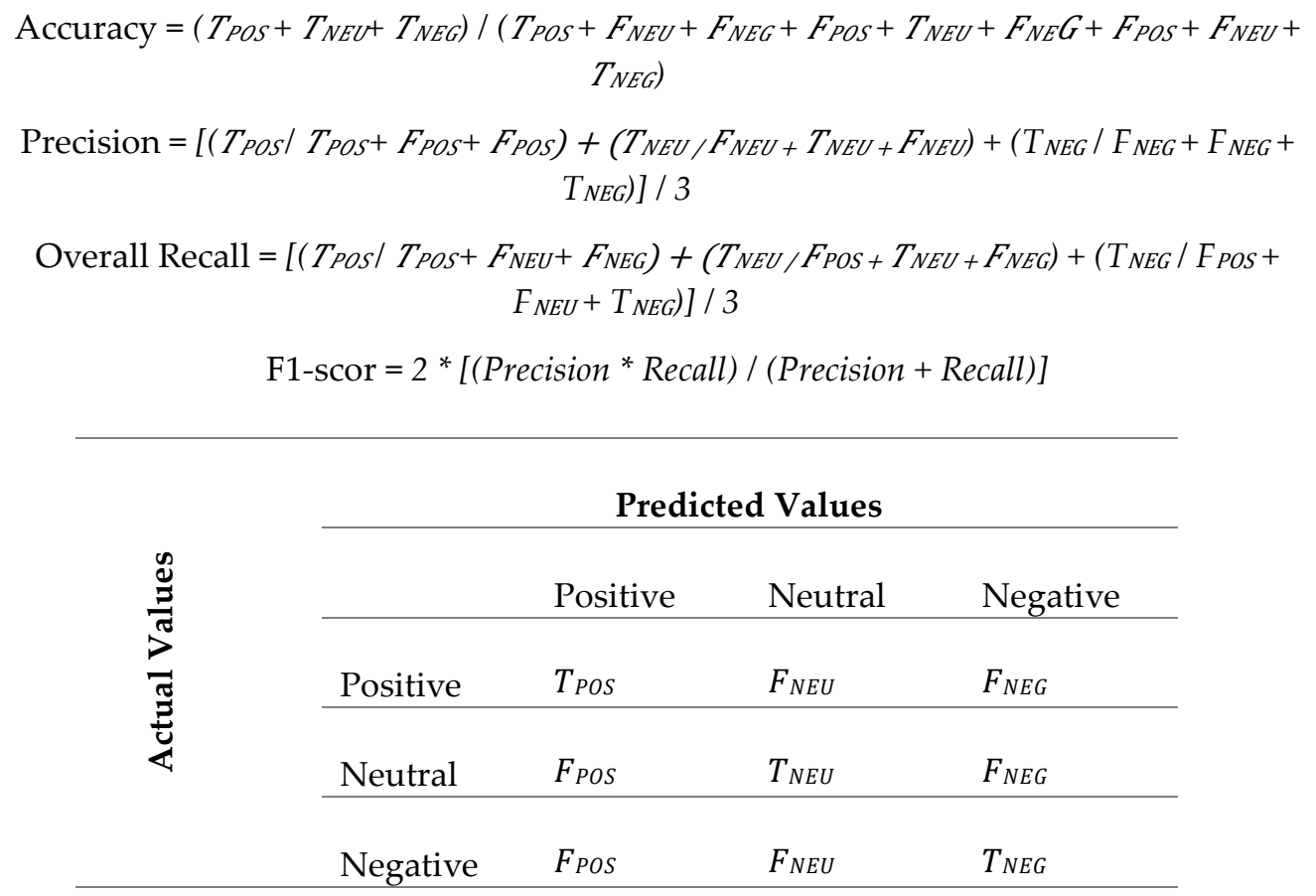

Figure 2. Confusion Matrix

\section{Results}

\subsection{Azure ML Logistic Regression Algorithm SA Model}

Figure 3 shows the results of Azure-based predictive model based on Logistic Regression for the dataset sentiments for tweets. The total number of tweets used to test the model of this experiment is 900 . It can be inferred that most of the sentiments expressed in the tweets were neutral with total of 348 . The total number of positive tweets were 289 and the number of negative tweets were 263. Table 1 shows the confusion Matrix for the dataset based on Logistics Regression Algorithm with the four evaluation metrices used for this study. As shown in Table 1, the Overall Recall value for the model tested on Azure ML using Logistics Regression algorithm is 0.557 . Moreover, the value of Overall Precision of the Azure ML model is 0.554. As for the value of Overall Accuracy, it is calculated as 0.560. Finally, the F1-score Value for this Azure ML model is 0.556. 


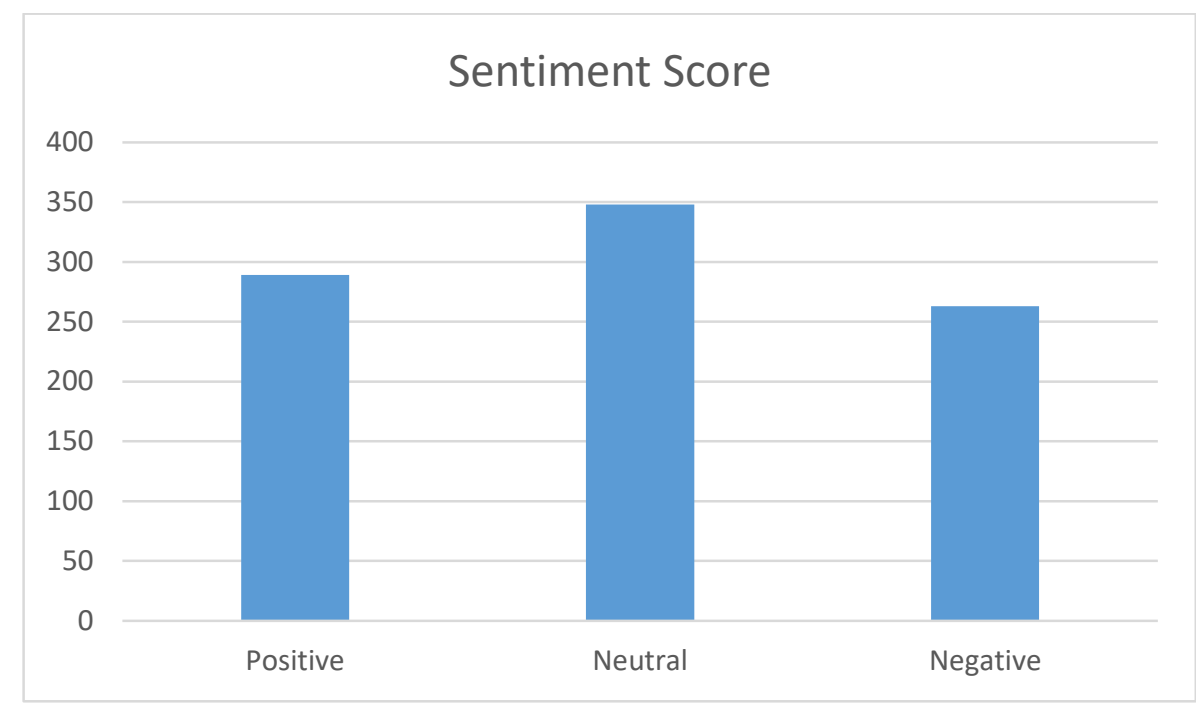

Figure 3. Azure SA Model's Results based on Logistic Regression

Table 1. Confusion Matrix based on Azure ML Logistic Regression Algorithm

\begin{tabular}{|c|c|c|c|c|}
\hline \multirow{6}{*}{ Actual Class } & \multicolumn{4}{|c|}{ Predicted Classes } \\
\hline & & Positive & Neutral & Negative \\
\hline & Positive & 139 & 78 & 57 \\
\hline & Neutral & 93 & 224 & 65 \\
\hline & Negative & 57 & 46 & 141 \\
\hline & \multicolumn{4}{|c|}{ Evaluation Metrics } \\
\hline Overall Recall & \multicolumn{4}{|l|}{0.557} \\
\hline Overall Precision & \multicolumn{4}{|l|}{0.554} \\
\hline Overall Accuracy & \multicolumn{4}{|l|}{0.560} \\
\hline F1-Score & \multicolumn{4}{|l|}{0.556} \\
\hline
\end{tabular}

\subsection{Amazon ML Logistic Regression Algorithm SA Model}

As shown in Figure 4, the results of Amazon-based predictive model based on Logistic Regression for the dataset sentiments for tweets. It can be inferred that most of the sentiments expressed in the tweets were negative with total of 368. The total number of positive tweets were 335 and the number of neutral tweets were 197. Table 2 shows the confusion Matrix for the dataset based on Logistics Regression Algorithm with the four evaluation metrices used for this study. The total number of tweets used to test the model of this experiment is 900. As shown in Table 2, the Overall Recall value for the model tested on Azure ML using Logistics Regression algorithm is 0.513. Moreover, the value of Overall Precision of the Azure ML model is 0.527. As for the value of Overall Accuracy, it is calculated as 0.497. Finally, the F1-score Value for this Azure ML model is 0.519. 


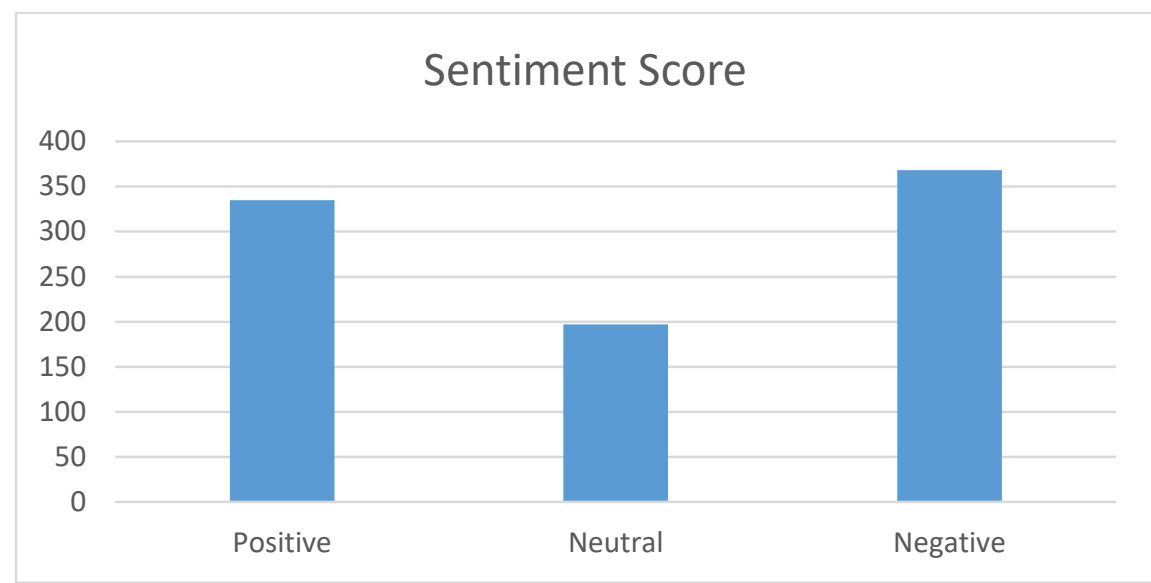

Figure 4. Amazon SA Model's Results based on Logistic Regression

Table 2. Confusion Matrix based on Amazon ML Logistic Regression Algorithm

\begin{tabular}{|l|l|c|c|c|}
\hline \multirow{4}{*}{ Actual Class } & \multicolumn{4}{|c|}{ Predicted Classes } \\
\cline { 2 - 5 } & & Positive & Neutral & Negative \\
\cline { 2 - 5 } & Positive & 145 & 33 & 74 \\
\cline { 2 - 5 } & Neutral & 101 & 139 & 131 \\
\cline { 2 - 5 } & Negative & 89 & 25 & 163 \\
\cline { 2 - 5 } & \multicolumn{4}{|c}{ Evaluation Metrics } \\
\hline Overall Recall & 0.513 & \multicolumn{4}{|}{} \\
\hline Overall Precision & 0.527 & \multicolumn{4}{|}{} \\
\hline Overall Accuracy & 0.497 & 0.519 & \\
\hline F1-Score & &
\end{tabular}

\subsection{Platforms and Models' Comparison}

Comparisons were made between SA predictive models build on Amazon and Azure ML considering the four studied factors; F-score, precision, recall and accuracy. As can be inferred from Figure 5, Microsoft Azure ML's F1-scores, Accuracy, Precision and Recall scores for dataset with values of $0.556,0.560,0.554$ and 0.557 consecutively were consistently greater than those of Amazon (F1-score: 0.519, Overall Accuracy: 0.497, Overall Precision: 0.527 and Overall Recall: 0.513). This demonstrates that SA models build using Logistic Regression on Microsoft Azure ML are more accurate and precise than SA models build using Logistic Regression on Amazon ML.

Moreover, the Azure ML interface is a graphical drag and drop interface and different modules can be selected to be included in the model by dragging those modules in the interface and based on the researchers' experiences, this ML platform is considered easier to use rather that the Amazon ML platforms in which users have no full control over their models since Amazon ML make most of the decisions. Furthermore, Azure ML supports quite a few data sources to train models such as CSV files, TXT files or even online data while Amazon ML platform has access only to data stored in Amazon cloud storages. Another comparison between both platforms is that in Azure ML users have the full control over which algorithm to utilize in building the model and can adjust the algorithm parameters based on their requirement. However, in Amazon ML the case is more restricted. 


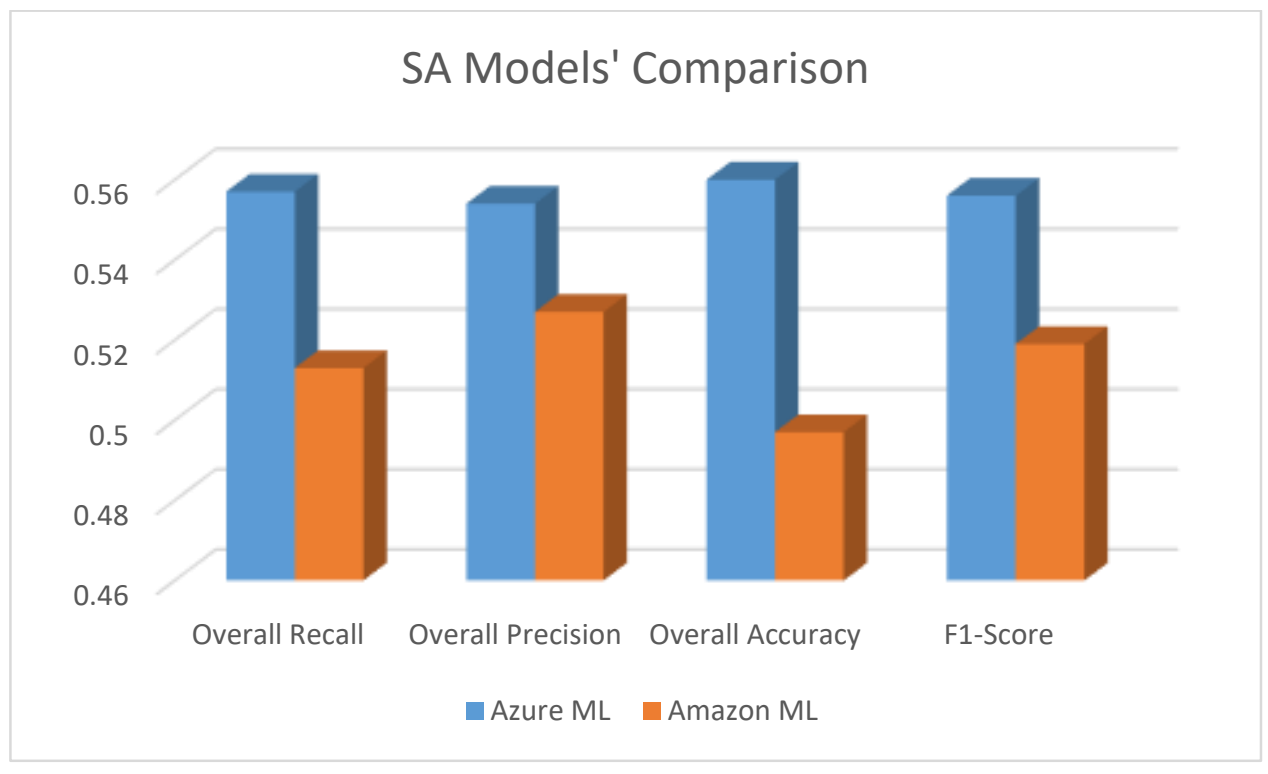

Figure 5. SA Models' Comparison

\section{Discussion}

For making the comparison between Azure ML and Amazon ML coherent, the comparison is divided into four main criterions; Interface, Data acquisition, Runtime and ML algorithms. Concerning the first criterion, the interface, in general and from the researchers' experiences in this study, Azure ML offers a collaborating interface, whereas Amazon ML offers a basic interface with limited features available to use. In particular, the Azure ML interface is a graphical drag and drop interface, unlimited numbers of modules can be selected to be included in the model by dragging those modules in the interface. Also, these modules can be connected together to generate a flow of steps that the model should follow to produce the results. On the other hand, the Amazon ML interface consists of a number of wizards that user use for creating models. The wizards make the process of creating these models uncomplicated, but in spite of that, users do not have full control over their models since Amazon ML make most of the decisions. The second criterion is Data Acquisition; Azure ML supports quite a few data sources to train models, firstly, training data can be uploaded from local files such as CSV file or a plain text (.txt) file. Secondly, online data that is located on a web URL that could be accessed via HTTP. Conversely, unlike Azure ML, Amazon ML has access only to data stored in Amazon storages including S3, Redshift and Amazon RDS. On other words, training data must be uploaded first into Amazon data storage before the initial process of building the model. The third criterion is ML Algorithms; Azure ML supports a wide range of ML algorithms in the area of classification, regression, and clustering. Other ML algorithms that are not supported by Azure ML can be added via code in R or Python. Furthermore, users have the full control over which algorithm to utilize in building the model and can adjust the algorithm parameters based on their requirement. However, in Amazon ML the case is different. Although Amazon ML is used to build models for classification and regression, users do not have control over which algorithm to use when building the model since Amazon ML support one algorithm for classification and one algorithm for regression, but users can change certain parameters for these algorithms.

The last criterion is Runtime; runtime is the time needed for each one of the classification models built over Azure ML and Amazon ML to predict the sentiments. This study evaluated the accuracy and precision of SA models build on Amazon and Azure machine learnings based on logistic regression analysis. SA models build of Azure platform had higher F-scores, accuracy, precision and recall scores than SA models build on Amazon 
platform. Results of the evaluation revealed that SA models build on Microsoft Azure ML based on Logistic Regression are more accurate and precise than SA models build on Amazon ML based on Logistic Regression. These results confirm the accuracy and precision of SA model build on Microsoft Azure ML platform and confirm that cloud-based ML platforms can be used to build SA models that can be used to analyse sentiments on social media networks such as twitter and Facebook. Result of this study has not been replicated elsewhere as no study has been conducted to compare the accuracy and precision of these SA models build on these two machine learnings. However, it has been acknowledged in several studies that SA models can be implemented on Azure and Amazon ML platforms [16], [17], [18] and [19]. For example, [20] compared sentiment classification models build on Azure platform to analyse the accuracy of Logistic Regression and Neural Network Algorithms. Reference [20] demonstrated that classification models build on these two machine learning algorithms yields equal level of accuracy in classifying financial tweets.

\section{Conclusion and Summary}

SA is playing a major technique for businesses in specific to identify individuals' opinions about their services and products. Thus, there is growing in the number of research in SA area. This research focused in the issue of building SA model based on cloud-based ML. In general, this research involved one main objective, which led to answer the research question: What ML platforms is better to provide reliable and accurate Sentiment Analysis?

This research explored the main characteristic of two main cloud-based ML platforms; Azure ML and Amazon ML. In general, Azure ML provides users with various tools to help in building the models and users have more control over their models. Conversely, Amazon ML offers users with limited tools and control as most of the choices in the model is determined by Amazon ML platform.

As demonstrated herein, Microsoft Azure machine offers the best platform for building SA models for analysis of social media sentiments. It is more accurate and precise than Amazon ML and can provide a more reliable SA models. Azure also offers multiple tools that can be used to simplify the process of creating classification models. It allows endusers to have more control on their SA models. This suggests that companies can leverage Microsoft Azure ML to undertake topic modelling detect customer sentiment. This can be done using state-of-art learning logistic regression algorithm.

Even though this research has achieved the objective specified previously, there is additional work that can be done to enhance the results of this research or to provide additional results based on different cloud-based ML platforms. Additional datasets that include more than a thousand records could be used to test the SA models built on Azure ML and Amazon ML. Moreover, since there are other cloud-based ML platforms, future research can compare both the platforms mentioned in this report with another platform such as IBM Watson Analytics.

The main limitation undertaking this study was the time constraints. Since checking the SA models results took around 8 hours for each of the 500 records to validate the results. Besides which, the tests were repeated for two reasons, firstly, to make sure the models are getting the same results from the same input, secondly, to record the time each model requires to execute. Therefore, under these circumstances, datasets used in testing the models was by, some mean small; the greatest one included 500 records.

Concerning benefits to the industry, this research could help businesses when determining which cloud-based ML platform to use in case they need to build SA models and to distinguish between the ML algorithms used in creating these models. As well as having a fully understanding on how cloud-based ML platforms work. 


\section{References}

[1] L. Quaisi and I. Aljarah, "A twitter Sentiment Analysis for Cloud Providers: A Case Study of Azure vs. AWS", 7th International Conference on Computer Science and Information Technology (CSIT), (2017) August 5-7.

[2] J. Du, J. Xu, H. Song and C. Tao, "Leveraging machine learning-based approaches to assess human papillomavirus vaccination sentiment trends with Twitter data”, MC Med Inform Decis Mak.., vol. 17, no. 2, (2017), pp. 69.

[3] A. Lin, "Improved Twitter Sentiment Analysis Using Naive Bayes and Custom Language Model", eprint arXiv:1711.1108, (2017).

[4] I. D. Addo, D. Do, R. Ge and I. A. Ahamed, "Reference Architecture for Social Media Intelligence Applications in the Cloud", IEEE 39th Annual Computer Software and Applications Conference, (2015) January 2-4.

[5] B. Liu, J. Zhang, Q. Liu, H. Li, M. Zhang, R. Qiu and J. Zhao, "Data Acquisition Hot Issues and System of Microblog Mining", Network and Information Systems for Computers 2015 International Conference on IEEE, doi: 10.1109/ICNISC.2015.89, (2015), pp. 116-119.

[6] L. Quaisi and L. Aljarah, "A twitter Sentiment Analysis for Cloud Providers: A Case Study of Azure vs. AWS", 7th International Conference on Computer Science and Information Technology (CSIT), (2017) May 6-8.

[7] T. Nasukawa and J. Yi, "Sentiment analysis: capturing favorability using natural language processing", In Proceedings of the 2nd international conference on Knowledge capture, Florida, USA, (2003) October 23-25, pp. 70-77.

[8] S. Morinaga, K. Yamanishi, K. Tateishi and T. Fukushima, "Mining product reputations on the web", In Proceedings of the eighth ACM SIGKDD international conference on Knowledge discovery and data mining, (2002), pp. 341-349.

[9] B. Pang, L. Lee and S. Vaithyanathan, "Thumbs up? Sentiment Classification using Machine Learning Techniques", Proc. of 7th EMNLP, (2002), pp. 79-86.

[10] A. D'Andrea, F. Ferri, P. Grifoni and T. Tiziana, "Approaches, Tools and Applications for Sentiment Analysis Implementation", International Journal of Computer Applications (0975-8887), vol. 25, no. 3, (2015).

[11] T. Hardeniya and D. A. Borikar, "An Approach to Sentiment Analysis Using Lexicons with Comparative Analysis of Different Techniques", IOSR Journal of Computer Engineering (IOSR-JCE) e-ISSN: 22780661, p-ISSN: 2278-8727, vol. 18, no. 3, ver. I, (2016) May-June, pp. 53-57.

[12] O. Harfoushi, D. Hasan and R. Obiedat, "Sentiment Analysis Algorithms through Azure Machine Learning: Analysis and Comparison”, Modern Applied Science, vol. 12, no. 7, (2018), pp. 49-58.

[13] M. Qasem, R. Thulasiram and P. Thulasiram, "Twitter sentiment classification using machine learning techniques for stock markets", Advances in Computing, Communications and Informatics (ICACCI), India, IEEE, (2015) May 7-11, pp. 834-840.

[14] O. Harfoushi, D. Hasan and R. Obiedat, "Sentiment Analysis Algorithms through Azure Machine Learning: Analysis and Comparison”, Modern Applied Science, vol. 12, no. 7, (2018), pp. 49-58.

[15] O. Harfoushi, D. Hasan and R. Obiedat, "Sentiment Analysis Algorithms through Azure Machine Learning: Analysis and Comparison", Modern Applied Science, vol. 12, no. 7, (2018), pp. 49-58.

[16] A. T. Bornstein, M. H. McLoughlin, J. Aguilar, W. S. Wong and B. D. Solomon, "Tracking medical genetic literature through machine learning", Molecular Genetics and Metabolism, vol. 118, no. 4, (2016), pp. 255-258.

[17] E. Mulholland, P. M. Kevitt, T. Lunney, J. Farren and J. Wilson, "360-MAM-Affect: Sentiment analysis with the Google prediction API and EmoSenticNet", Intelligent Technologies for Interactive Entertainment (INTETAIN), 7th International Conference, (2015) June 7-12, pp. 217-221.

[18] M. Bihis and S. Roychowdhury, "A generalized flow for multi-class and binary classification tasks: An Azure ML approach”, Big Data (Big Data), 2015 IEEE International Conference, Santa Clara: IEEE, (2015) January 3-7, pp. 1728-1737.

[19] S. Roychowdhury, "Classification of Large-Scale Fundus Image Data Sets: A Cloud-Computing Framework", Proceedings of the Annual International Conference of the IEEE Engineering in Medicine and Biology Society, Lake Buena Vista: IEEE, (2016) March 5-8, pp. 3256-3259.

[20] M. Qasem, R. Thulasiram and P. Thulasiram, "Twitter sentiment classification using machine learning techniques for stock markets", Advances in Computing, Communications and Informatics (ICACCI), India: IEEE, (2015) May 7-11, pp. 834-840.

[21] D. Hasan, "A Performance Analysis of Cloud-Based Sentiment Analysis Using Machine Learning”, A dissertation submitted in partial fulfilment of the requirements of Sheffield Hallam University, (2017). 
International Journal of Advanced Science and Technology

Vol.118 (2018) 